\title{
SOLPS-ITER simulations of the TCV divertor upgrade
}

M. Wensing ${ }^{1}$, B. P. Duval ${ }^{1}$, O. Février ${ }^{1}$, A. Fil ${ }^{2}$, D. Galassi ${ }^{1}$, E. Havlickova ${ }^{1}$, A. Perek $^{3}$, H. Reimerdes ${ }^{1}$, C. Theiler ${ }^{1}$, K. Verhaegh ${ }^{1,2}$, M. Wischmeier ${ }^{4}$, the EUROfusion MST1 team ${ }^{5}$ and the TCV team ${ }^{6}$

${ }^{1}$ Ecole Polytechnique Fédérale de Lausanne (EPFL), Swiss Plasma Center (SPC), CH-1015 Lausanne, Switzerland

${ }^{2}$ York Plasma Institute, Department of Physics, University of York, Heslington, York, YO105DD, United Kingdom

${ }^{3}$ DIFFER - Dutch Institute for Fundamental Energy Research, De Zaale 20, 5612 AJ Eindhoven, The Netherlands

${ }^{4}$ Max Planck Institut für Plasmaphysik, Boltzmannstr. 2, 85748 Garching, Germany

${ }^{5}$ See the author list of H. Meyer et al., Nucl. Fusion 57 (2017) 102014

${ }^{6}$ See the author list of S. Coda et al., Nucl. Fusion 57 (2017) 102011

E-mail: mirko.wensing@epfl.ch

Submitted to: Plasma Phys. Control. Fusion 
Abstract. The effect of the upcoming TCV divertor upgrade on the distribution of neutrals and the onset of detachment is studied using 2D transport code simulations. The divertor upgrade is centered around the installation of a gas baffle to form a divertor chamber of variable closure. SOLPS-ITER simulations predict that the baffle geometry selected to be installed in TCV in 2019 increases the divertor neutral density by a factor $\sim 5$ and the neutral compression by one order of magnitude in typical TCV single null, Ohmic heated scenarios $(330 \mathrm{~kW})$. The compression increases further with the addition of auxiliary heating systems (1.2 MW). Simulations show that volumetric power losses in the divertor increase giving access to deeper detachment for given upstream densities and heating power. Predictions for observations by various TCV diagnostics, including baratrons, divertor spectrometer and visible camera systems, are presented to guide the experimental verification of the efficiency of the divertor baffles.

Keywords: magnetic fusion, TCV, SOLPS, plasma-wall interaction, detachment

\section{Introduction}

Dissipative divertors, as required for ITER and the future demonstration power plant DEMO, rely amongst other mechanisms on a transfer of energy and momentum from charged particles to neutrals. Presently, the recycling neutrals in the TCV divertor can directly transit from the divertor targets to the main plasma. This flow of neutrals to the main plasma decreases the divertor neutral density and hence the volumetric power losses in the divertor. An in-vessel gas baffle will be installed during the upcoming TCV-divertor upgrade with the aim of extending the available scenarios to regimes of greater relevance for future fusion devices [1]. The baffle will be located at $z \approx-0.35 \mathrm{~m}$ separating the vessel into two regions, referred to as main chamber and divertor chamber in the following. A scan of the baffle size using the SOLPS-ITER edge transport code predicts that a baffle which limits the SOL at a flux surface with a separatrix distance of $3-4 \lambda_{q}$ yields maximal neutral compression [2]. This optimum baffle size, which is interpreted as trade-off between divertor closure and main chamber recycling on the baffle tiles, has guided the design of the baffle that will be installed in the first phase of the upgrade [3].

The installation of the gas baffles will be accompanied by various diagnostic upgrades including an improved Langmuir probe coverage, a tangential infrared camera system, additional bolometer channels, additional chords for the divertor spectrometer system (DSS), a new multispectral imaging system (MANTIS), divertor Thomson scattering and a new set of magnetic probes [1][2].

This paper examines the performance of the predicted optimal baffle length in terms of the resulting neutral distribution and detachment behavior using the transport code SOLPS-ITER [4][5]. The sensitivity of various diagnostics to monitor the expected divertor conditions is discussed. In particular, predictions for pressure measurements (baratrons), ionisation and CIII emissivity front movement as well as Balmer line intensities (DSS [6]/MANTIS [7]) are presented. The article is structured as follows. The simulation setup is described in section 2. The effect of baffles on plasma and neutral density evolution during density ramps is discussed in section 3 , followed by predictions for baratron and spectroscopic measurements in section 4. Conclusions are presented in section 5 .

\section{Simulation setup}

SOLPS-ITER (B2.5-Eirene) is a transport code that couples the B2.5 multi-fluid solver with the kinetic Monte Carlo model neutral code Eirene [4][5]. At present, this code is one 
of the best regarded tools for simulation of the scrape-off layer and has guided the design of the ITER divertor [8]. The results presented herein are obtained using the SOLPS-ITER version 3.000.006.

\subsection{B2.5}

The B2.5 code solves multi-fluid balance equations of particle, parallel momentum and energy for every fluid species $\alpha$. For this study, the simulations include deuterium as main plasma species together with carbon that is the main impurity in TCV: $\alpha=\left\{\mathrm{D}^{+}, \mathrm{C}^{+}\right.$, $\left.\mathrm{C}^{++}, \ldots, \mathrm{C}^{6+}\right\}$. The radial heat and particle transport is assumed anomalous and incorporated by introducing cross-field diffusion with species-independent, spatially constant transport parameters $D_{\perp, \alpha}=0.2 \mathrm{~m}^{2} \mathrm{~s}^{-1}$ and $\chi_{\perp, \alpha}=$ $1.0 \mathrm{~m}^{2} \mathrm{~s}^{-1}$, as chosen in previous simulations [2]. These values yield typically measured radial upstream density and temperature falloff lengths $\left(\lambda_{n}, \lambda_{T} \sim 1 \mathrm{~cm}\right)$ on TCV. The fieldaligned quadrangular simulation grid is shown in Figure 1a) with a radial extent limited by the inner baffle. This results in a distance of the B2.5 grid to the outer baffle of $5 \mathrm{~mm}$. Radial flow of charged particles beyond the farSOL boundary is converted to neutrals and passed to the kinetic neutral model. This limitation of the model may lead to an overestimation of the particle source in the main chamber. In the simulated cases, the number of particles passed into the main chamber by this crossfield flow is however $\leq 10 \%$ of those introduced by gas puffing. Future simulations with the SolEdge2D code [9] will aim to review this constraint and better evaluate the main chamber recycling flux [10]. Heating power is provided over the radial core boundary in the closed flux surface region as Neumann boundary condition to the energy equation and is equally dis- tributed to electrons and ions. Drift effects are not included in the present study. The effect of drifts on detachment has been demonstrated to give rise to bifurcation and redistribution of power and particles between the target plates [11]. This should not affect strongly the trends observed during the presented scans but may affect the absolute values.

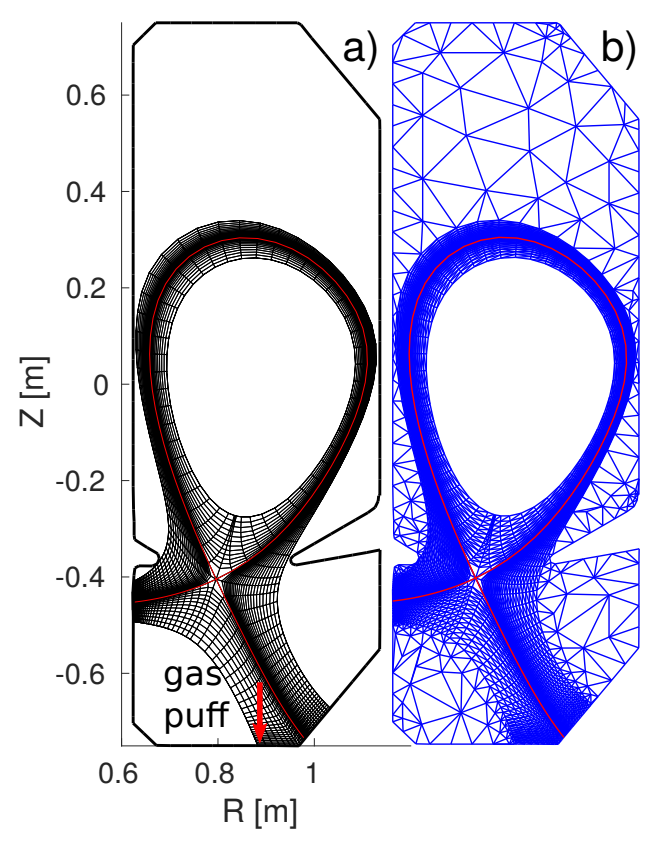

Figure 1. a) B2-grid, b) Eirene grid.

For the two divertor configurations, with and without gas baffles, a range of upstream densities (from $0.5 \cdot 10^{19} \mathrm{~m}^{-3}$ to $4 \cdot 10^{19} \mathrm{~m}^{-3}$ ) and input powers of $330 \mathrm{~kW}$ and $1.2 \mathrm{MW}$ are explored to study the evolution of detachment during TCV density ramps in discharges with Ohmic and auxiliary heating, respectively.

\subsection{Eirene}

The Eirene code describes the neutral particle distribution by a kinetic Monte-Carlo approach. It allows to include atomic and molecular reactions as well as surface interactions in the simulation. Such a kinetic treatment is crucial for an accurate description once the 
neutral mean-free path becomes big compared to the spatial extent of the SOL, which is usually the case in TCV where the neutral meanfree path can be several centimeters even in the region containing closed field lines. Neutralplasma reactions are summarized in Table 1, with the corresponding reaction rates dependent on the local plasma density and temperature in each fluid cell. Hence, no reactions between neutrals and plasma take place outside the fluid grid. Since the mean-free path of neutral self-collisions is of the order meters in the divertor volume, neutral-neutral collisions are disabled in the simulation to save computation time. In the baffled case with high heating power and highest density the self-collision mean free path reaches $0.1 \mathrm{~m}$ locally at the outer strike point and is hence still large compared to the scrape-off layer width. The neutral densities and pressures are computed by averaging particle positions over the triangular mesh depicted in Figure 1b). Due to numerical issues encountered at low temperatures, $T_{e}$ and $T_{i}$ values passed to Eirene are constrained to be larger than $0.5 \mathrm{eV}$. This limits the volumetric recombination rate close to the targets in deeply detached cases but is not expected to affect the main conclusions presented herein.

The model introduces deuterium molecules via a gas puff at coordinates close to the experimental location $(R=0.880 \mathrm{~m}, Z=$ $-0.748 \mathrm{~m})$. The puff is varied to obtain a specified electron separatrix density at the outer midplane.

TCV experiments indicate that the particle balance in the vessel during discharges is barely affected by the turbo pumps, indicating that the sink of particles is dominated by the graphite wall tiles. In the simulation this is accounted for by introducing a constant recycling coefficient of 0.99 over the entire wall. Thus, gas puff rates were obtained that agree

\begin{tabular}{|c|c|}
\hline \multicolumn{2}{|c|}{ AMJUEL } \\
\hline $\mathrm{D}+\mathrm{e} \rightarrow \mathrm{D}^{+}+2 \mathrm{e}$ & H.4, H.10 2.1.5 \\
\hline $\mathrm{e}+\mathrm{C} \rightarrow C^{+}+2 \mathrm{e}$ & H.4, H.10 2.6A0 \\
\hline $\mathrm{e}+\mathrm{D}_{2} \rightarrow 2 e+\mathrm{D}_{2}^{+}$ & H.4 2.2 .9 \\
\hline $\mathrm{e}+\mathrm{D}_{2} \rightarrow e+\mathrm{D}+\mathrm{D}$ & H.4 $2.2 .5 \mathrm{~g}$ \\
\hline $\mathrm{e}+\mathrm{D}_{2} \rightarrow 2 e+\mathrm{D}+\mathrm{D}^{+}$ & H.4 2.2.10 \\
\hline $\mathrm{D}^{+}+\mathrm{D}_{2} \rightarrow \mathrm{D}^{+}+\mathrm{D}_{2}$ & H.0, H.1, H.3 0.3T \\
\hline $\mathrm{D}^{+}+\mathrm{D}_{2} \rightarrow \mathrm{D}+\mathrm{D}_{2}^{+}$ & H.2 3.2 .3 \\
\hline $\mathrm{e}+\mathrm{D}_{2}^{+} \rightarrow \mathrm{e}+\mathrm{D}+\mathrm{D}^{+}$ & H.4 2.2.12 \\
\hline $\mathrm{e}+\mathrm{D}_{2}^{+} \rightarrow 2 \mathrm{e}+\mathrm{D}^{+}+\mathrm{D}^{+}$ & H.4 2.2.11 \\
\hline $\mathrm{e}+\mathrm{D}_{2}^{+} \rightarrow \mathrm{D}+\mathrm{D}$ & H.4, H.8 2.2.14 \\
\hline $\mathrm{D}^{+}+\mathrm{e} \rightarrow \mathrm{D}(1 \mathrm{~s})$ & H.4, H.10 2.1.8 \\
\hline \multicolumn{2}{|c|}{ HYDHEL } \\
\hline $\mathrm{D}^{+}+\mathrm{D}(1 \mathrm{~s}) \rightarrow \mathrm{D}(1 \mathrm{~s})+\mathrm{D}^{+}$ & H.1, H.3 3.1.8 \\
\hline \multicolumn{2}{|c|}{ ADAS } \\
\hline $\mathrm{e}+\mathrm{C}^{+} \rightarrow \mathrm{C}+h \nu$ & $\begin{array}{l}\text { H. } 4 \text { acd } 96 \\
\text { H.10 prb96 }\end{array}$ \\
\hline
\end{tabular}

Table 1. Reactions included in the simulations with the respective numbers in the databases.

with the experimental rates to within a factor of 2 (Table 2). It is interesting to note that the puff rates are a factor of $\sim 2-4$ higher with baffles installed to maintain identical upstream densities. This is a consequence of the increased particle flux to the target plates and the increased collision rates with wall elements by neutrals due to the higher density in the baffled divertor region. Wall reflection of hydrogen atoms leads to molecular association and thermalization with the wall temperature $T_{\text {wall }}=300 \mathrm{~K}$.

Carbon impurities result from machine wall (near complete coverage of the first wall with graphite protection tiles) sputtering. Physical sputtering, according to the RothBogdansky formula, and chemical sputtering with a yield of $3.5 \%$ are used [12]. This yield was verified by quantitative comparison between the simulated CIII (465 nm) intensity, obtained through a synthethic diagnostic, with 
absolutely calibrated measurements on TCV using the DSS absolutely calibrated visible spectroscopy system. These choices lead to volume averaged $\left\langle Z_{\text {eff }}\right\rangle=1.1-2.0$, depending on input power and upstream density $\left(\left\langle Z_{e f f}\right\rangle\right.$ increases with $P_{\text {core }}$ and decreases with $\left.n_{e}^{u}\right)$.

\section{Predictions for the baffled TCV divertor}

Dissipative divertors, as required for future fusion reactors, will need to operate in a detached state where the target heat loads are mitigated to meet the material constraints of plasma-facing components. The installation of gas baffles aims to improve access to such deeper detachment and enabling operation in regimes of higher relevance for a fusion reactor by increasing the divertor neutral density and, thus, enhancing the volumetric power losses. Here we investigate the evolution of the simulated plasma and neutral distribution with and without baffles during an upstream density ramp.

\subsection{Neutral distribution}

The neutral density $n_{\text {neut }}=n_{D^{0}}+2 n_{D_{2}}$ is evaluated on the triangular Eirene mesh. With the baffles installed, the average main chamber neutral density $\left\langle n_{\text {neut }}\right\rangle_{\text {main }}$ is reduced by factor of $\sim 2-3$ (Figure $2 \mathrm{~b}$ ), the corresponding divertor neutral density $\left\langle n_{\text {neut }}\right\rangle_{\text {div }}$ increases by a factor of $\sim 5$ (Figure 2c). The neutral compression (Fig 2d) is here defined as

$c_{D} \equiv\left\langle n_{\text {neut }}\right\rangle_{\text {div }} /\left\langle n_{\text {neut }}\right\rangle_{\text {main }}$.

The compression increases by an order of magnitude with the baffles and shows further increase (factor $\sim 3$ ) with heating power increased from $330 \mathrm{~kW}$ to $1.2 \mathrm{MW}$. The enhancement of compression with heating power results from a shorter ionisation meanfree path $\lambda_{i o n}^{m f p}=v_{n} /\left(n_{e}\left\langle\sigma_{i o n} v_{e}\right\rangle\right)$ of $\mathrm{D}^{0}$ in the vicinity of the baffle opening. The mean-free path decreases with $n_{e}$ and $T_{e}$ (at temperatures below $\sim 150 \mathrm{eV}$ which is the relevant regime for the TCV divertor), which both increase with $P_{\text {core }}$ (Figure $3 \mathrm{a}$ ). The dissociation meanfree path for $\mathrm{D}_{2}$ molecules and the ionisation mean-free path for $\mathrm{C}^{0}$ atoms are much smaller compared to the ionisation mean-free path of $\mathrm{D}^{0}$ (Figure $3 \mathrm{~b}$ ). The divertor neutral confinement is therefore dominated by the ionisation efficiency of atomic deuterium $\mathrm{D}^{0}$ in the vicinity of the baffle opening and the separatrix.

Figure 4 shows the neutral density along the divertor tiles numbered in the poloidal clock-wise direction. The highest neutral density, unsurprisingly, is found at the inner and outer strike points, where recycling is dominant. Here the increase in neutral density with the baffles is smallest $(\sim 3)$ but increases to $\sim 5-10$ after a few centimeters away. Here the neutral density, mainly governed by molecules, stays approximately constant. A secondary neutral density spike in the private flux region is related to the gas puff location $(R=0.88 \mathrm{~m})$. The simulated fast fall-off in neutral pressure from the strike points appears to be in qualitative agreement with present experiments where the neutral pressure, measured at a single point in the divertor using a baratron, decreases quickly as the distance between strike point and baratron location is increased [13]. The experimental evaluation of the neutral compression, as defined in equation 1 , is difficult to interpret as it often relies on few local pressure measurements. Future advances in spectroscopic analysis may provide volumetric information about atomic neutral densities as various hydrogenic line intensities 


\begin{tabular}{c|c|c|c|c|c|c|c|c} 
upstream density & $0.5 \mathrm{e} 19$ & $1 \mathrm{e} 19$ & $1.5 \mathrm{e} 19$ & $2 \mathrm{e} 19$ & $2.5 \mathrm{e} 19$ & $3 \mathrm{e} 19$ & $3.5 \mathrm{e} 19$ & $4 \mathrm{e} 19$ \\
\hline no baffle $(330 \mathrm{~kW})$ & $7.7 \mathrm{e} 19$ & $1.9 \mathrm{e} 20$ & $3.5 \mathrm{e} 20$ & $5.5 \mathrm{e} 20$ & $7.9 \mathrm{e} 20$ & $1.0 \mathrm{e} 21$ & $1.3 \mathrm{e} 21$ & $1.5 \mathrm{e} 21$ \\
baffle $(330 \mathrm{~kW})$ & $1.5 \mathrm{e} 20$ & $4.5 \mathrm{e} 20$ & $1.0 \mathrm{e} 21$ & $1.8 \mathrm{e} 21$ & $2.6 \mathrm{e} 21$ & $5.0 \mathrm{e} 21$ & $/$ & $/$ \\
\hline no baffle $(1.2 \mathrm{MW})$ & $1.2 \mathrm{e} 20$ & $2.3 \mathrm{e} 20$ & $4.5 \mathrm{e} 20$ & $9.2 \mathrm{e} 20$ & $1.8 \mathrm{e} 21$ & $2.8 \mathrm{e} 21$ & $3.7 \mathrm{e} 21$ & $4.5 \mathrm{e} 21$ \\
baffle $(1.2 \mathrm{MW})$ & $2.0 \mathrm{e} 20$ & $5.7 \mathrm{e} 20$ & $2.4 \mathrm{e} 21$ & $4.6 \mathrm{e} 21$ & $7.1 \mathrm{e} 21$ & $9.8 \mathrm{e} 21$ & $1.3 \mathrm{e} 22$ & $/$
\end{tabular}

Table 2. Deuterium puffing rate $\left[\right.$ atoms/s] needed to maintain the given upstream density $\left[\mathrm{m}^{-3}\right]$ in the simulation, experimentally the gas puff is $\approx 1 \cdot 10^{20} \mathrm{~s}^{-1}$ to reach an upstream density of $\approx 1 \cdot 10^{19} \mathrm{~m}^{-3}$ (\#52312) and $\approx 2 \cdot 10^{21} \mathrm{~s}^{-1}$ to reach $n^{u} \approx 4 \cdot 10^{19} \mathrm{~m}^{-3}(\# 62591)$.

depend linearly upon it.

\subsection{Upstream and target plasma parameters}

It is expected that the increasing upstream density leads to significant temperature gradients along field lines as the collisionality increases with density. This is generally thought to be concurrent with (often quadratic) increases in the ion target current during the attached phase. A deviation, i.e. flattening and eventual roll-over, of the ion target current from this trend is taken to be an indicator for detachment.

The simulations show that, with increasing upstream density, the upstream temperature decreases such that the upstream electron pressure is approximately conserved (Figure 5a), i.e. approximately $T_{e}^{o m p, s e p} \propto 1 / n_{e}^{o m p, s e p}$. Also experimentally, we observe a decrease of upstream temperature with increasing upstream density. Multiple effects could contribute to the decrease of $T_{e}^{o m p, s e p}$, such as a decrease of the target temperature an increased parallel convective transport, upstream radiation losses and widening of the SOL. The outer target density increases (Figure 5b) with the upstream density, leading to a reduction of the ionisation-mean free path and an enhanced ionisation. When power limitation results, i.e. when the power entering the recycling region is insufficient to completely ionise the incoming neutral flux, the ionisation rate saturates and so the electron density stops increasing. As the collisionality increases, a parallel temperature gradient develops due to finite parallel heat conductivity. Additional volumetric power losses associated with hydrogenic ionisation and impurity radiation further decrease temperature at the target (Figure 5c,d). According to the simulation, hydrogenic ionisation and impurity radiation contribute approximately equally to the total volumetric losses in the divertor. The increase in the target current with upstream density is weaker than quadratic (Figure 5e), consistent with observations in TCV experiments during detached divertor conditions [13]. The further flattening, and eventual roll-over, of the target current is again attributed to the saturation of the ionisation particle source due to power limitation/starvation. The power reaching the target is thus reduced as consequence of increasing volumetric losses as the upstream density increases.

With the installation of the gas baffles, we expect to access deeper detachment conditions as the increased divertor neutral density enhances hydrogenic excitation and ionisation losses as well as molecular dissociation. Unless impurity radiation dominates power loss in the divertor, which is not the case in the simulation, the presence of the baffle should have a significant impact on the divertor conditions. 

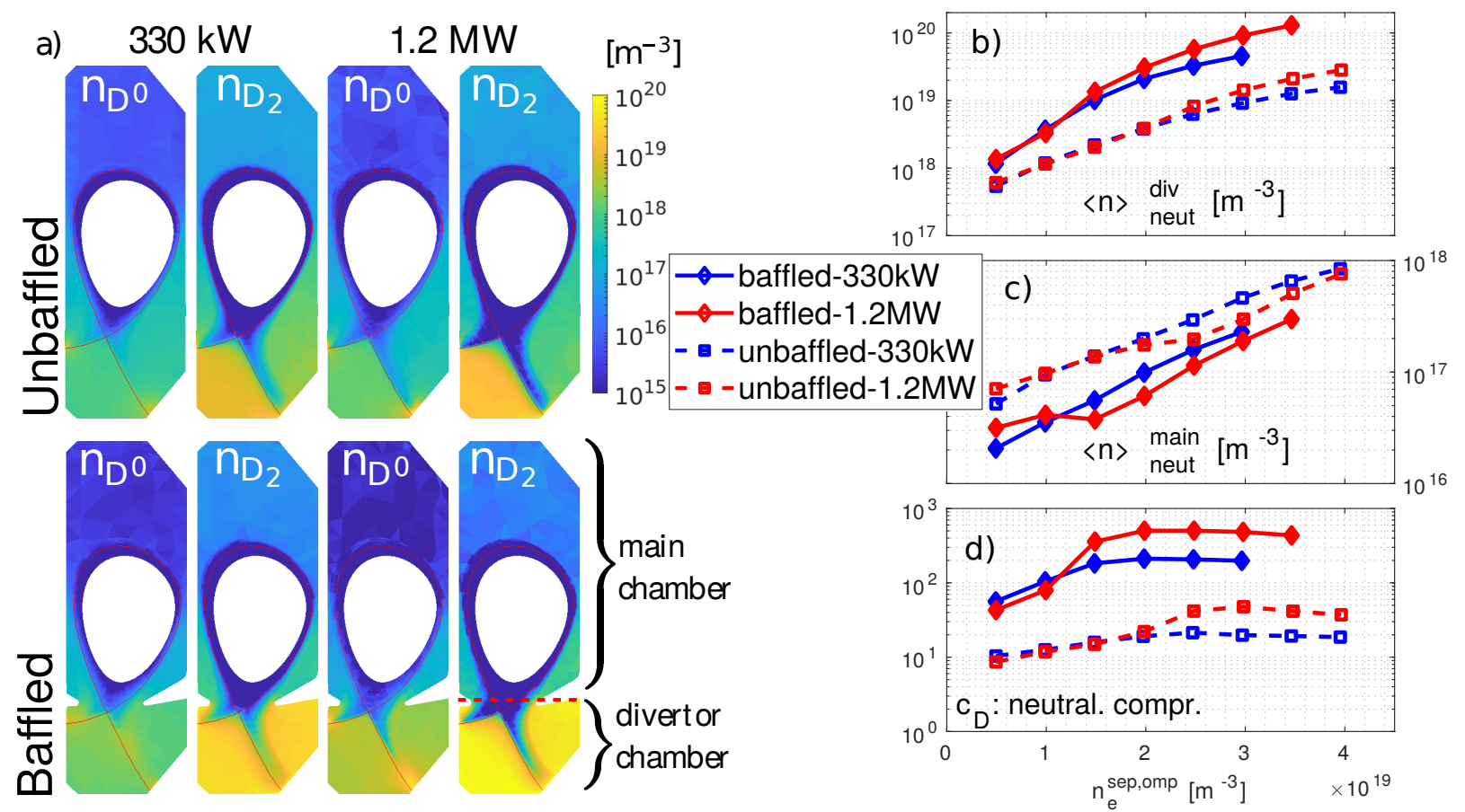

Figure 2. a) atomic and molecular deuterium neutral distribution at $n_{e}^{\text {sep,omp }}=2.5 \cdot 10^{19} \mathrm{~m}^{-3}$, b) volume averaged neutral density in the divertor, c) main chamber, d) resulting neutral compression.
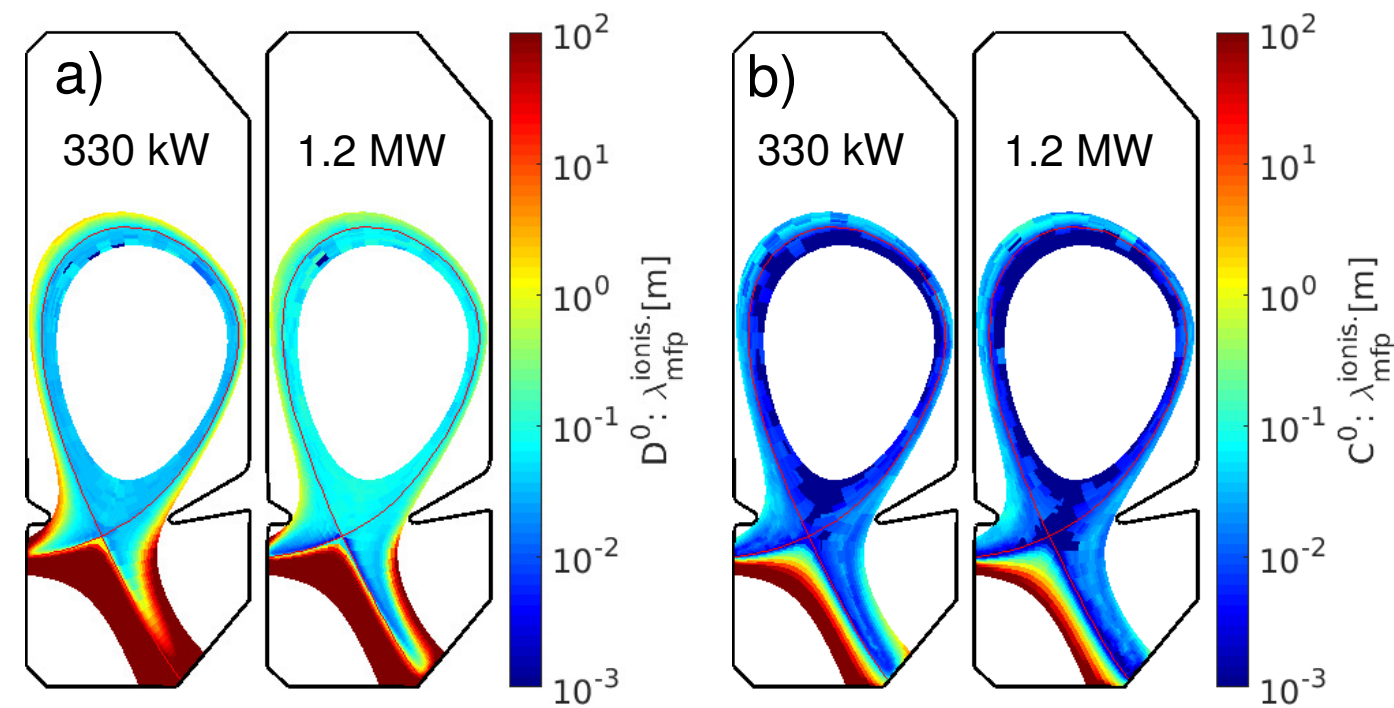

Figure 3. Atomic deuterium (a) and carbon (b) ionisation mean-free path at $330 \mathrm{~kW}$ and $1.2 \mathrm{MW}$ heating power at $n_{e}^{\text {sep,omp }}=2.5 \cdot 10^{19} \mathrm{~m}^{-3}$

The simulation of the baffled/unbaffled divertor shows an upstream temperature unaffected by the presence of baffles indicating that properties of the main plasma are largely unaffected by the baffle (Figure 5a). The outer target density increases (Figure 5b), due to an increase in neutral density that leads to a reduction of the ionisation-mean free path and, thus, an enhanced ionisation. The flattening, and eventual roll-over, of the outer target ion 


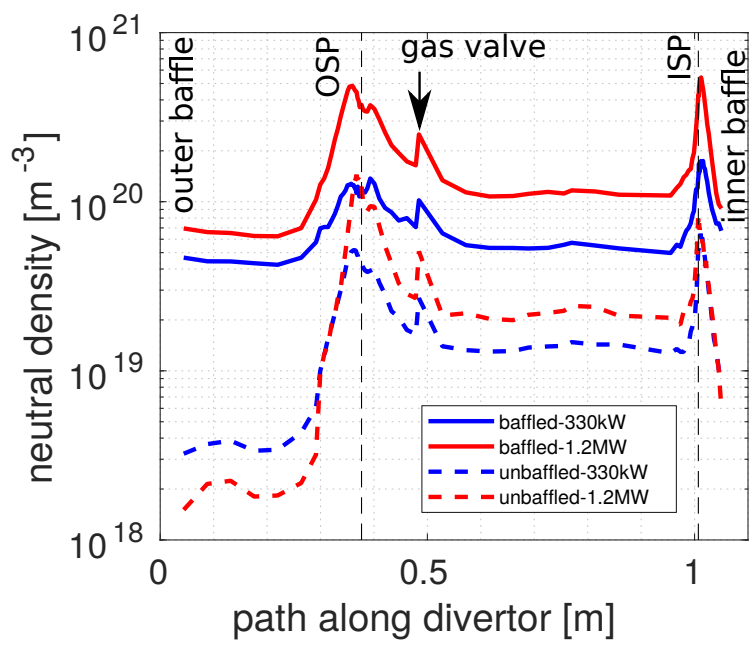

Figure 4. Neutral density along the divertor tiles in the region below $\mathrm{z}=-0.4 \mathrm{~m}$ unwrapped in clockwise direction, dashed lines correspond to strike point positions, $n_{e}^{\text {sep,omp }}=2.5 \cdot 10^{19} \mathrm{~m}^{-3}$

current commences at lower upstream densities $\left(\Delta n_{e}^{\text {sep,omp }} \sim 1 \cdot 10^{19} \mathrm{~m}^{-3}\right.$, Figure 5e) due to saturation of the ionisation source at lower upstream density. The power to the outer target is significantly reduced as consequence of the increased volumetric losses (Figure 5f).

In summary, the presence of the baffle and the accompanied increase in neutral density leads to a positive feedback of increasing electron density, decreasing ionisation meanfree path and thus increasing ionisation source. As more power is used for ionisation, the target temperature decreases, leading to an earlier onset of detachment as power limitation commences at a lower upstream density. The curves that include the baffles resemble the unbaffled cases but shifted by $\Delta n_{e}^{\text {sep,omp }} \sim$ $1 \cdot 10^{19} \mathrm{~m}^{-3}$, indicating that similar divertor conditions are achieved at lower upstream density.

\subsection{Ionisation front movement}

One of the indicators for plasma cooling during density ramp experiments is the displacement
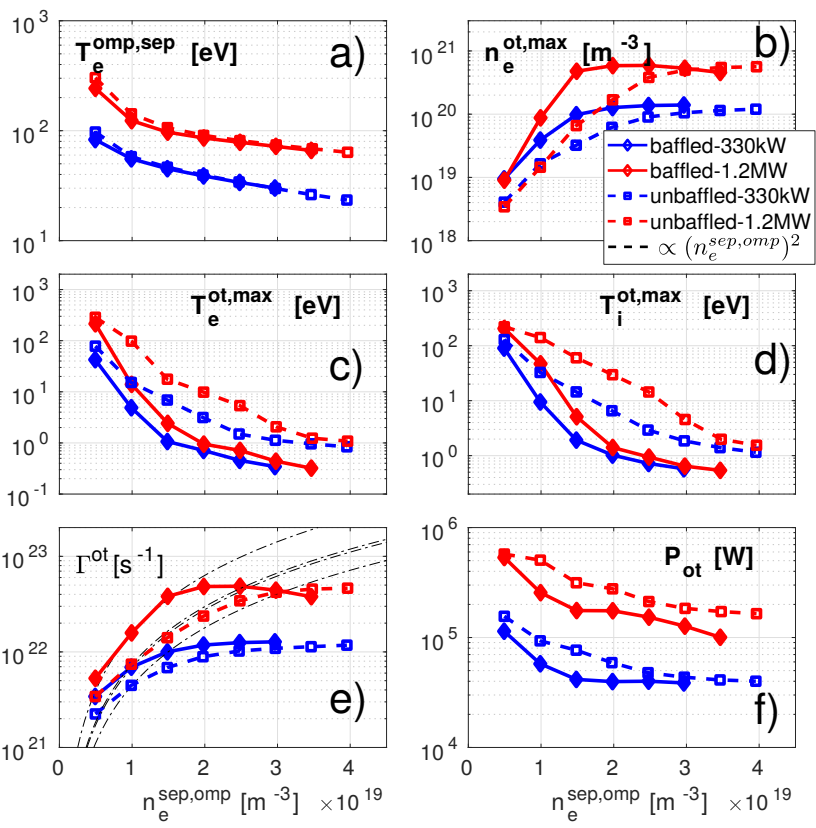

Figure 5. Variation of upstream and target quantities with upstream density. Solid lines=baffled, dashed=unbaffled, red $=1.2 \mathrm{MW} \quad$ (hot case) and blue $=330 \mathrm{~kW}$ (cold case)

of the ionisation fronts towards the X-point, as a consequence of saturation of the ionisation source[14, Fig. 11]. This displacement is also observed in simulations (Figure 6a)[15]. Herein, the term front is defined as follows: the poloidal location for which $90 \%$ of the total ionisation takes place in the region between it and the X-point. The simulated ionisation front position correlates with a local electron temperature of $T_{e}^{\text {front }}=4 \mathrm{eV}$ (Figure 6b). This reduction can be explained by the strong reduction of the probability of an ionisation event below $T_{e}<5 \mathrm{eV}$. As the ionisation region recedes from the target, the neutral density and the ionisation mean free path increase below the ionisation region (Figure $3 \mathrm{a}$ and Figure 2 a).

As baffles are included in the simulation, the ionisation front position is located at greater distance to the target for given upstream density (Figure 6b) as a result of the 
access to deeper detachment as discussed in Section 3.2.

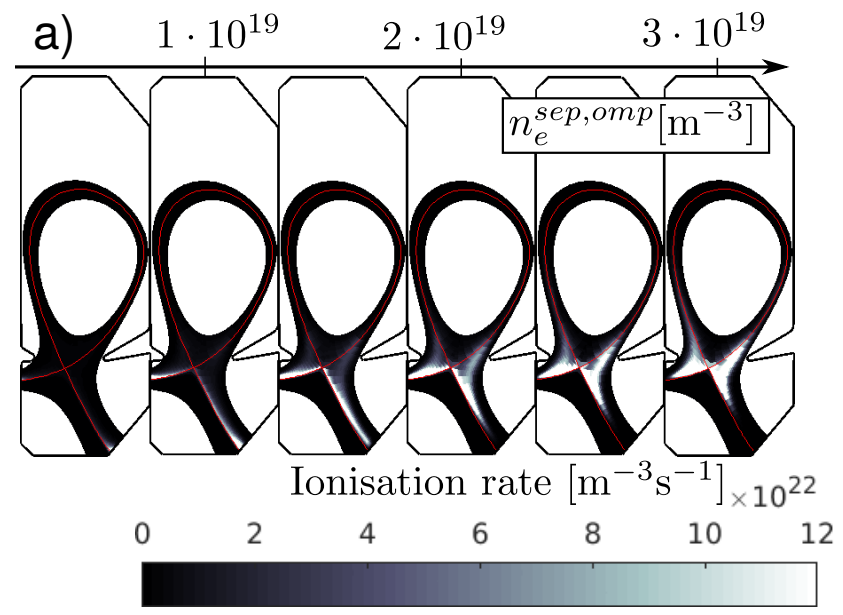

ionisation front: distance from out. target [m]

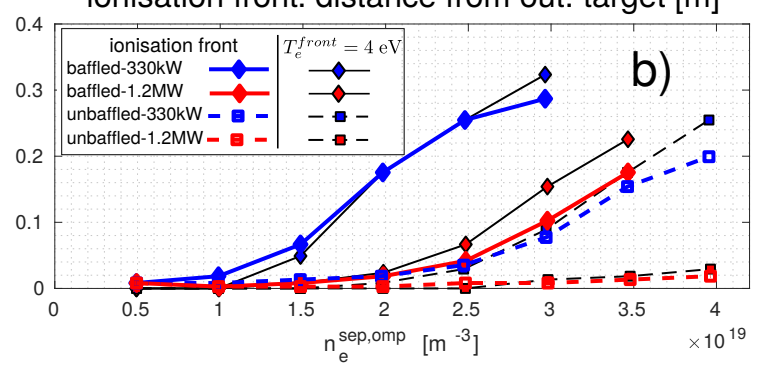

Figure 6. a) Ionisation front movement with varying upstream density for the case with baffle and $330 \mathrm{~kW}$ heating power, b) distance of the ionisation and $T_{e}=$ $4 \mathrm{eV}$-front from the outer target.

\section{Synthetic diagnostics}

The effectiveness of the baffles will be evaluated experimentally using localized neutral pressure (baratrons) and spectroscopic measurements (MANTIS/DSS). The interpretation of which is often not directly linked to the neutral content but convoluted with various physical mechanisms. Based on the simulations, predictions for the upcoming experiments are presented to guide the future experiments.

\subsection{Baratron}

To experimentally compare the effectiveness of the baffled and the unbaffled TCV divertor, the neutral pressure in the main chamber and divertor volume will be monitored using magnetically shielded and vibrationally isolated baratron pressure gauges similar to those used on JET [16]. Two such pressure gauges are presently installed on the outer wall (at $Z=0 \mathrm{~m}$ ) and the floor (at $R=0.75 \mathrm{~m}$ ). They are mounted on the end of tubes to help shield the gauge from the tokamak magnetic field [13]. The neutral pressure is expected to drop along the tube due to association of atoms and thermalization with the wall making these measurements hard to interpret in absolute term.

A model for the translation of neutral parameters at the vessel edge $\left(n^{0}, p^{0}\right)$ to the corresponding parameters seen at the baratron gauge $\left(n^{\text {gauge }}, p^{\text {gauge }}\right)$ is needed to relate the $2 \mathrm{D}$ simulation results to experimental measurements. Such a zero dimensional model has been proposed by Niemczewski [17]. It assumes a molecular flow $\left(\lambda_{m f p} \gg\right.$ $D$ ) where $D$ is the diameter of the tube, conservation of flux, thermalization of particles and association of atoms to molecules upon wall contact.

$n_{\text {mol }}^{\text {gauge }}=\frac{n_{\text {atom }}^{0}}{\sqrt{2}} \sqrt{\frac{T_{\text {atom }}^{0}}{T_{\text {wall }}}}+n_{\text {mol }}^{0} \sqrt{\frac{T_{\text {mol }}^{0}}{T_{\text {wall }}}}$
$p_{\text {gauge }}=n_{\text {mol }}^{\text {gauge }} T_{\text {wall }}$

The baratron pressure is of the order of $10 \mathrm{mPa}$ for typical TCV conditions. Assuming a wall temperature of $T_{\text {wall }}=300 \mathrm{~K}$ in the baratron tube and a complete thermalization of neutrals, we have a molecular self-collision mean-free path of $\lambda_{m f p, c o l l}=$ $k_{B} T_{\text {wall }} /\left(\sqrt{2} \pi p_{\text {mol }} d_{m}^{2}\right) \approx 15 \mathrm{~m}$ where $p_{\text {mol }}$ is the molecular pressure and $d_{m}=74 \mathrm{pm}$ is the 
diameter of a hydrogen molecule [18], justifying the molecular flow assumption. The validity of the 0D-model is further discussed in the appendix, where comparison to Eirene for a simplified baratron geometry is presented.

The simulated pressure and temperature profiles are averaged over the circular tube opening of the baratron. These values at the tube entrance can then be related to the gauge pressure using the 0D-model to estimate the measurements following baffle installation on TCV (Figure 7). For the unbaffled case, experiments at $n_{e}^{\text {sep,omp }} \approx 1 \cdot 10^{19} \mathrm{~m}^{-3}$ (\#52312) yield baratron measurements of 3 and $6 \mathrm{mPa}$ at the midplane and at the divertor, respectively. For comparable upstream density SOLPS-ITER (corrected with equations 2, 3) yields 3 and $12 \mathrm{mPa}$, respectively. For the high density case $n_{e}^{\text {sep,omp }} \approx 4 \cdot 10^{19} \mathrm{~m}^{-3}(\# 62591)$ the agreement between experimental $\left(p_{d i v}=\right.$ $50 \mathrm{mPa}, p_{\text {mid }}=5 \mathrm{mPa}$ ) and simulated pressure $\left(p_{\text {div }}=140 \mathrm{mPa}, p_{\text {mid }}=20 \mathrm{mPa}\right)$ is worse. The pressure trends and the orders of magnitudes are, however, well reproduced.

With the baffles, the pressure at the divertor gauge is predicted to increase by $\sim 2-5$ depending on density and heating power (Figure 7), while the pressure in the midplane baratron will drop by a factor $\sim$ $2-3$. These changes at the baratrons are significant and should be readily measurable. However, the pressure at the midplane gauge drops to values below the present dynamic range of the pressure gauges $(1.3 \mathrm{mPa}-2.7 \mathrm{~Pa})$. Quantitative estimates of the experimental neutral compression based on present pressure gauges may therefore be difficult. It would be desirable to increase the diagnostic sensitivity to lower pressures.

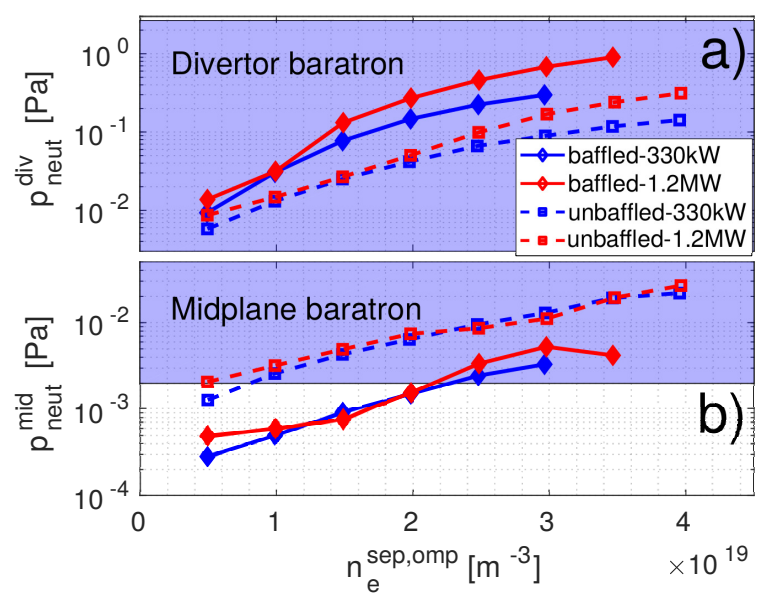

Figure 7. Synthetic baratrons after correction with the 0D-model. Areas shaded in blue indicate the present dynamic range of the TCV baratron $(1.3 \mathrm{mPa}-$ 2.7 Pa).

\subsection{Spectroscopy}

Information about the divertor state can also be deduced by observing line radiation as emission is sensitive to plasma and neutral parameters in the divertor. The recently installed multispectral imaging system MANTIS enables simultaneous observation of a large set of spectrally filtered images, corresponding to certain emission lines, using narrow bandpass filters to obtain poloidal radiation maps of single transition lines [19]. The divertor spectrometer system DSS enables quantitative measurement of radiation along single lines of sight in the divertor volume [6].

\subsubsection{Balmer line emission As visible} Balmer lines depend on the neutral deuterium density, it is of interest to estimate the variation of Balmer emission signal strength with the baffles. Atomic radiative emission occurs with de-excitation of atomic electrons through electron-neutral (excitation) and electrion-ion (volumetric recombination) collisions. The emission for each process is evaluated by us- 
ing the photon emission coefficients from the ADAS database [20] based on the plasma conditions calculated by SOLPS. Reassuringly, the radiated power calculated agrees to within a few percent with the radiated power directly calculated by SOLPS.

The volume integrated intensity of Balmer lines increases by factor $\sim 3-10$ with the baffles (Figure 8). This can be attributed to the simultaneous increase of electron and neutral particle densities. The least intense Balmer line, routinely observed with MANTIS corresponds to the Deuterium $n=7 \rightarrow 2$ transition. Simulations indicate that higher transitions in the series such as $n=9 \rightarrow 2$ should give detectable intensities. After including the wavelength-dependent absorption and reflectivity of the optical components and camera sensor sensitivity, the signal strength for baffled discharges will be comparable to the experimentally observable signal of the $7 \rightarrow 2$ line in the present, unbaffled, conditions. Higher-n Balmer lines are especially useful as indicator of volumetric recombination and in inferring the characteristic temperature for the recombination region using a Boltzmann fit [21].

Simulations show that all Balmer line emission is most intense near the target plates for attached conditions. As the upstream density increases, excitation emission becomes dominant closer to the X-point, while recombination is only significant at very low temperatures $(\sim 1 \mathrm{eV})$, i.e. close to the targets. The higher Balmer line intensities then become increasingly recombination dominated. At high densities, this leads to a poloidal separation of the hot, excitation-dominated radiation region and the cold, recombination-dominated, radiation region. This effect can be observed in the lower Balmer lines $\left(D_{\alpha}\right.$ and $\left.D_{\beta}\right)$ showing a poloidal separation of the emission regions (Figure 9), while the higher Balmer line emis- sion remains close to the target plates. The poloidal separation has also been observed experimentally in TCV discharges using the DSS in conditions where it was possible to seperate the excitation and recombination contributions of the Balmer lines [14].

The simulations suggest that with baffles the spatial separation of low Balmer lines should be visible at significantly lower upstream densities. Emission rates calculated from ADAS contain only the atomic contribution to the radiation. So, experimentally, this clear effect may be clouded by molecular emission which contributes mostly to the lower Balmer lines. Nevertheless, it can be hoped that such a clear separation of excitation and recombination regions can be observed in some region of the Balmer series.

\subsubsection{CIII-radition The CIII $(465 \mathrm{~nm})$ emis-} sion line front is often taken as measure of the local temperature of $\sim 5-8 \mathrm{eV}$ [13]. For a given upstream density, the CIII front, utilizing the definition given in section 3.3, shows significantly higher distance from the target plates between baffled and unbaffled cases (Figure 10). Hence, observation of front positions is shown to be a simple way of testing the simulation predictions and the efficiency of the gas baffles.

\section{Conclusions}

SOLPS-ITER simulations have been carried out to study the effect of the TCV divertor gas baffle on the divertor performance and to provide predictions for future experiments. The simulations predict an increase of the divertor neutral density by a factor $\sim 5$ and enhanced neutral compression by one order of magnitude. The neutral compression is further increased by a factor $\sim 3-4$ with heat- 


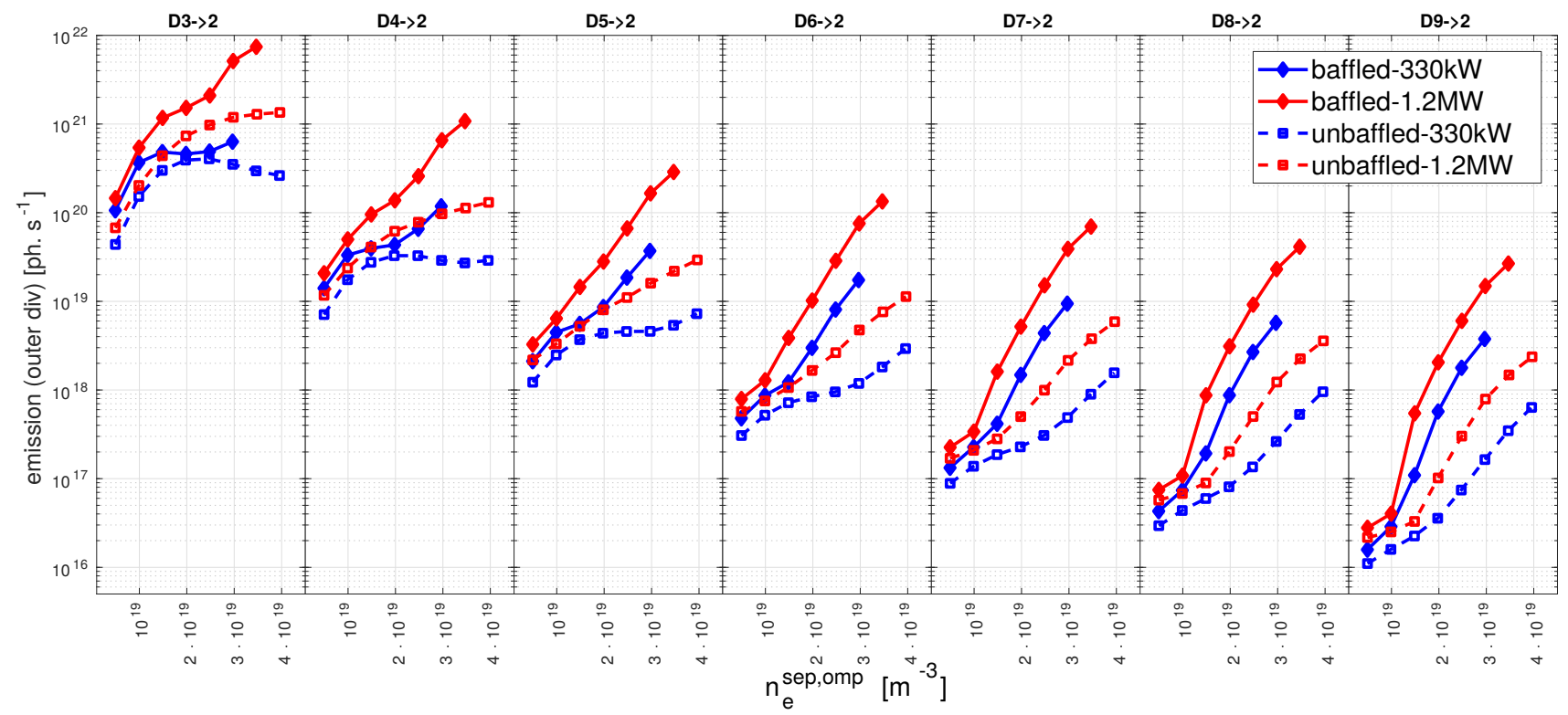

Figure 8. Spatially integrated Balmer line emission in the outer divertor up to $n=9$

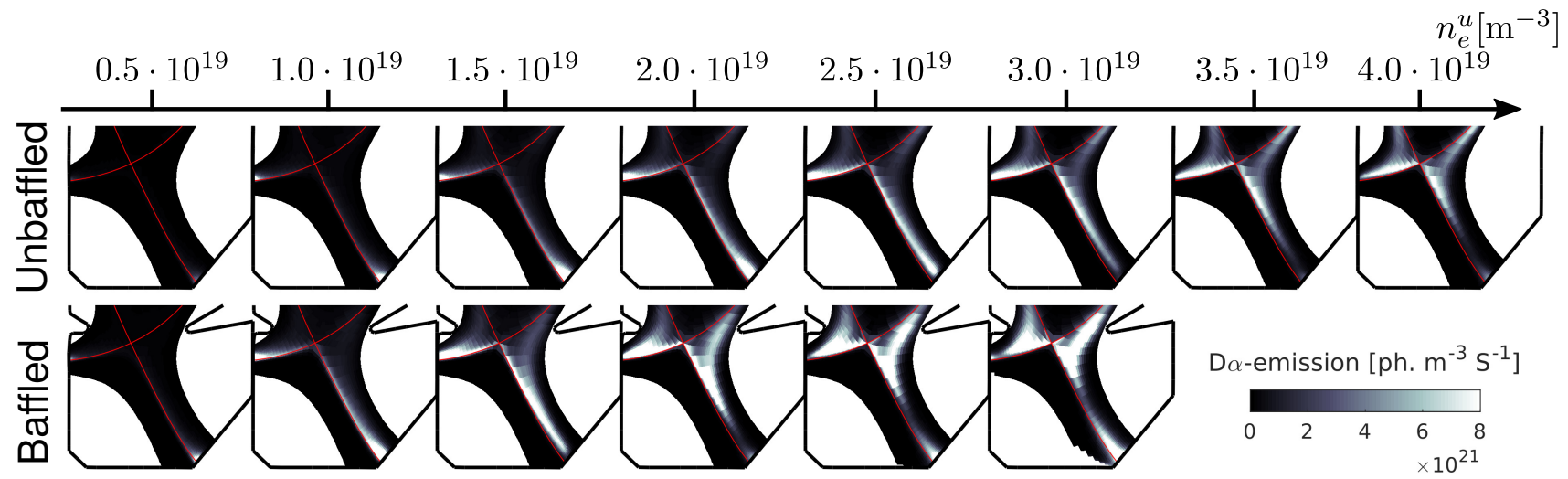

Figure 9. Spatial separation of atomic $\mathrm{D} \alpha$ emission due to separation of excitation and recombination regions $(330 \mathrm{~kW}$ case)

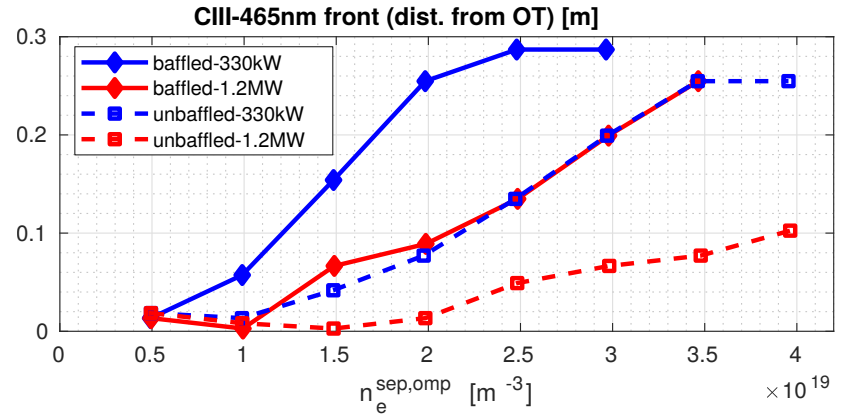

Figure 10. CIII-front distance from the outer target using the front definition given in section 3.3. ing power due to reduction in the ionisation mean-free path of $\mathrm{D}^{0}$ in the vicinity of the baffle opening. Facilitated access to detachment and to deeper detachment due to enhanced volumetric losses in the divertor volume are predicted. Clear spatial displacements of the ionisation and the experimentally measured CIII (465 nm) front are expected at lower upstream densities $\left(\Delta n \sim 10^{19} \mathrm{~m}^{-3}\right)$. With the gas baffle, this front position will provide an important indicator that is accessi- 
ble to presently installed available diagnostics (MANTIS/DSS). Synthetic baratrons for the midplane and the divertor position predict a relative signal change of factor $\sim 3$, suggesting that experimental probing of the effect of baffling is also accessible to baratron measurements. However, the midplane pressure is predicted to become affected strongly by sensitivity threshold of the baratron that may hinder a direct quantitative experimental measurement of the neutral compression. The future TCV operation with baffles will not only increase our knowledge on the main factor determining detachment but will also provide a direct test of the SOLPS-ITER model for initial ITER operation and beyond.

\section{Appendix}

In order to relate neutral densities and pressures in the simulation domain to the pressure measured by the baratron gauges a 0D-model is utilized in section 4.1. Comparison between the 0D-model (equations 2 and 3) and SOLPSITER is presented by means of a simulation with a tube as radial extension to the TCV vessel at $z=-0.4 \mathrm{~m}$ extending for $L \approx 2.7 \mathrm{~m}$ with diameter $D=5 \mathrm{~cm}$ and $T_{\text {wall }}=300 \mathrm{~K}$ (Figure A1a). Surface particle pumping is disabled on the wall segments of the tube. Note that, in such a two dimensional simulation, this setup describes a toroidally symmetric baratron. For a more realistic three dimensional tube the probability of wall collisions along a trajectory would increase and therefore yield a faster pressure drop along the longitudinal extension of the tube.

Energetic atomic neutrals flow into the baratron which are converted to thermal molecules upon wall contact. Along the extent of the tube, the atomic influx equals the outflux of molecules (Figure A1b), hence the total number of particles is conserved. The density of atomic neutrals decreases rapidly along the tube, whereas the molecular density remains constant after a distance of a few centimeters from the opening (Figure A1c). The total pressure decays quickly along the extent of the tube (Figure A1d) due to conversion of energetic atomic neutrals to thermal molecules. Despite the low density of atomic neutrals their contribution to the total pressure is significant. After the 90 degree bend, all atomic neutrals are converted to neutrals. Hence, gauge pressure is determined solely by wall temperature and molecular density at the gauge.

The simple 0D-model matches the Eirene estimate of pressure and density within a factor of $\sim 5 \%$ at the end of the baratron tube. Further tests of different baratron geometries consistently yielded satisfactory agreement $(\leq$ $20 \%$ deviation) between Eirene and the 0Dmodel unless the atomic contribution is nonnegligible, e.g. in shorter tubes without a bend in which the pressure gauge is in line of sight to the plasma and hence receives energetic neutrals.

The TCV midplane baratron gauge, located at $Z=0 \mathrm{~m}$, is mounted on the end of a straight tube with diameter of $D=4 \mathrm{~cm}$ with length $L=0.8 \mathrm{~m}$. The TCV divertor baratron tube is equal in diameter with $L=1.5 \mathrm{~m}$ and two consecutive $90^{\circ}$ bends. Hence for both baratron geometries sufficient number of neutral wall collisions are expected to justify the assumptions of the 0D model. The 2D simulations confirm that the $0 \mathrm{D}$ model is a good approximation of the TCV baratrons and can be used to compare measurements with simulations. 


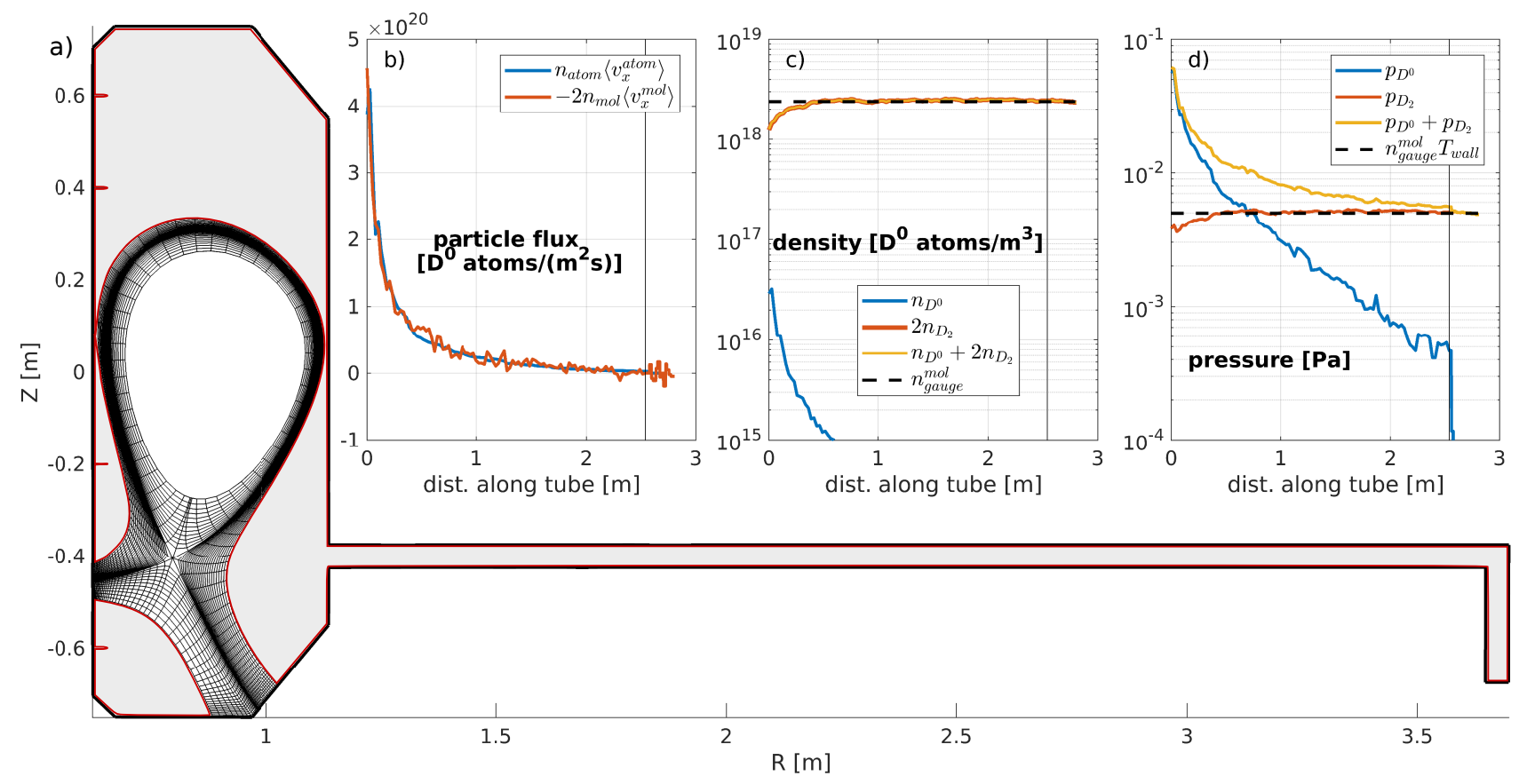

Figure A1. SOLPS-ITER estimate of the radial dependence of b) particle fluxes, c) densities and d) pressures along a simplified baratron tube with 90 degree bend (shown in a).

\section{Acknowledgments}

This work has been carried out within the framework of the EUROfusion Consortium and has received funding from the Euratom research and training programme 2014 - 2018 and 2019 - 2020 under grant agreement No 633053. The views and opinions expressed herein do not necessarily reflect those of the European Commission. This work was supported in part by the Swiss National Science Foundation.

[1] H. Reimerdes, et al., Nucl. Mat. En. 12, 11061111,2017

[2] A. Fasoli, TCV heating and divertor upgrade, 27th IAEA Fus. En. Conf., Gandhinagar, India, FIP/P8-6, 22-27 Oct. 2018

[3] D. Vaccaro, et al., Thermal, electromagnetic and structural analysis of gas baffles for the TCV divertor upgrade, submitted to Fusion Eng. Des.

[4] S. Wiesen, et al., J. Nucl. Mat. 463, 480-484, 2015

[5] X. Bonnin, et al., Plasma Fusion Res. 11,

\section{3, 2016}

[6] K. Verhaegh, et al., Nucl. Mat. En. 12, 11121117,2017

[7] W. Vijvers, at al., J. Instrum. 12, C12058, 2017

[8] A. Kukushkin, et al., Fusion Eng. Des. 86, 28652873, 2011

[9] H. Bufferand et al., Nucl. Fus. 55, 053025, 2015

[10] D. Gallassi et al., Performance simulation of divertor neutral baffles in the TCV tokamak with the SolEdge2D-EIRENE code, 60th APS Div. Plas. Phys., Portland, Oregon, USA, PP11.00027, 5-9 Nov. 2018

[11] A. Jaervinen, et al., Phys. Rev. Let. 121, 075001, 2018

[12] J. Davis, A. Haasz, Jour. Nucl. Mat. 241-243, 37-51, 1997

[13] C. Theiler et al., Nucl. Fus. 57, 072008, 2017

[14] K. Verhaegh, et al., An improved understanding of the roles of atomic processes and power balance in divertor target ion current loss during detachment, to be published. DOI: 10.13140/RG.2.2.24292.48005/1

[15] A. Fil, et al., Contrib. Plasm. Phys. 58, 746-750, 2018

[16] U. Kruezi, et al., Rev. Sci. Instrum. 83, 10D728, 2012

[17] A. Niemczewski, PhD thesis, MIT, Cambridge, 
1995

[18] C. Edelmann, Vakuumphysik, Spektrum Akademischer Verlag, Heidelberg/Berlin, S. 38, 1988

[19] A. Perek, Multispectral Advanced Narrowband Tokamak Imaging System, to be submitted to Rev. Sci. Instrum.

[20] H.P. Summers, The ADAS User Manual, version $2.6 \mathrm{http}: / /$ www.adas.ac.uk, 2004

[21] B. Lipschultz, et al., Phys. Plasmas 6, 1907, 1999 